


\section{Efficiently Computed Lexical Chains as an Intermediate Representation for Automatic Text Summarization}

\author{
H. Gregory Silber* \\ University of Delaware
}

\author{
Kathleen F. McCoy ${ }^{\dagger}$ \\ University of Delaware
}

While automatic text summarization is an area that has received a great deal of attention in recent research, the problem of efficiency in this task has not been frequently addressed. When the size and quantity of documents available on the Internet and from other sources are considered, the need for a highly efficient tool that produces usable summaries is clear. We present a linear-time algorithm for lexical chain computation. The algorithm makes lexical chains a computationally feasible candidate as an intermediate representation for automatic text summarization. A method for evaluating lexical chains as an intermediate step in summarization is also presented and carried out. Such an evaluation was heretofore not possible because of the computational complexity of previous lexical chains algorithms.

\section{Introduction}

The overall motivation for the research presented in this article is the development of a computationally efficient system to create summaries automatically. Summarization has been viewed as a two-step process. The first step is the extraction of important concepts from the source text by building an intermediate representation of some sort. The second step uses this intermediate representation to generate a summary (Sparck Jones 1993).

In the research presented here, we concentrate on the first step of the summarization process and follow Barzilay and Elhadad (1997) in employing lexical chains to extract important concepts from a document. We present a linear-time algorithm for lexical chain computation and offer an evaluation that indicates that such chains are a promising avenue of study as an intermediate representation in the summarization process.

Barzilay and Elhadad (1997) proposed lexical chains as an intermediate step in the text summarization process. Attempts to determine the benefit of this proposal have been faced with a number of difficulties. First, previous methods for computing lexical chains have either been manual (Morris and Hirst 1991) or automated, but with exponential efficiency (Hirst and St.-Onge 1997; Barzilay and Elhadad 1997). Because of this, computing lexical chains for documents of any reasonable size has been impossible. We present here an algorithm for computing lexical chains that is linear in space and time. This algorithm makes the computation of lexical chains computationally feasible even for large documents.

* Department of Computer and Information Sciences, Newark, DE 19711. E-mail: silber@udel.edu $\dagger$ Department of Computer and Information Sciences, Newark, DE 19711. E-mail: mccoy@mail.eecis. udel.edu 
A second difficulty faced in evaluating Barzilay and Elhadad's proposal is that it is a proposal for the first stage of the summarization process, and it is not clear how to evaluate this stage independent of the second stage of summarization. A second contribution of this article is a method for evaluating lexical chains as an intermediate representation. The intuition behind the method is as follows. The (strong) lexical chains in a document are intended to identify important (noun) concepts in the document. Our evaluation requires access to documents that have corresponding humangenerated summaries. We run our lexical chain algorithm both on the document and on the summary and examine (1) how many of the concepts from strong lexical chains in the document also occur in the summary and (2) how many of the (noun) concepts appearing in the summary are represented in strong lexical chains in the document.

Essentially, if lexical chains are a good intermediate representation for text summarization, we expect that concepts identified as important according to the lexical chains will be the concepts found in the summary. Our evaluation of 24 documents with summaries indicates that indeed lexical chains do appear to be a promising avenue of future research in text summarization.

\subsection{Description of Lexical Chains}

The concept of lexical chains was first introduced by Morris and Hirst. Basically, lexical chains exploit the cohesion among an arbitrary number of related words (Morris and Hirst 1991). Lexical chains can be computed in a source document by grouping (chaining) sets of words that are semantically related (i.e., have a sense flow). Identities, synonyms, and hypernyms/hyponyms (which together define a tree of "is a" relations between words) are the relations among words that might cause them to be grouped into the same lexical chain. Specifically, words may be grouped when:

- Two noun instances are identical, and are used in the same sense. (The house on the hill is large. The house is made of wood.)

- Two noun instances are used in the same sense (i.e., are synonyms). (The car is fast. My automobile is faster.)

- The senses of two noun instances have a hypernym/hyponym relation between them. (John owns a car. It is a Toyota.)

- The senses of two noun instances are siblings in the hypernym/hyponyn tree. (The truck is fast. The car is faster.)

In computing lexical chains, the noun instances must be grouped according to the above relations, but each noun instance must belong to exactly one lexical chain. There are several difficulties in determining which lexical chain a particular word instance should join. For instance, a particular noun instance may correspond to several different word senses, and thus the system must determine which sense to use (e.g., should a particular instance of "house" be interpreted as sense 1, dwelling, or sense 2, legislature). In addition, even if the word sense of an instance can be determined, it may be possible to group that instance into several different lexical chains because it may be related to words in different chains. For example, the word's sense may be identical to that of a word instance in one grouping while having a hypernym/hyponym relationship with that of a word instance in another. What must happen is that the words must be grouped in such a way that the overall grouping is optimal in that it creates the longest/strongest lexical chains. It is our contention that words are grouped into a single chain when they are "about" the same underlying concept. 


\section{Algorithm Definition}

We wish to extract lexical chains from a source document using the complete method that Barzilay and Elhadad implemented in exponential time, but to do so in linear time. Barzilay and Elhadad define an interpretation as a mapping of noun instances to specific senses, and further, of these senses to specific lexical chains. Each unique mapping is a particular "way of interpreting" the document, and the collection of all possible mappings defines all of the interpretations possible. In order to compute lexical chains in linear time, instead of computing every interpretation of a source document as Barzilay and Elhadad did, we create a structure that implicitly stores every interpretation without actually creating it, thus keeping both the space and time usage of the program linear. We then provide a method for finding that interpretation which is best from within this representation. As was the case with Barzilay and Elhadad, we rely on WordNet ${ }^{1}$ to provide sense possibilities for, and semantic relations among, word instances in the document.

Before we could actually compute the interpretations, one issue we had to tackle was the organization and speed of the WordNet dictionary. In order to provide expedient access to WordNet, we recompiled the noun database into a binary format and memory-mapped it so that it could be accessed as a large array, changing the WordNet sense numbers to match the array indexes.

\subsection{Chain Computation}

Before computation can begin, the system uses a part-of-speech tagger ${ }^{2}$ to find the noun instances within the document. Processing a document involves creating a large array of "metachains," the size of which is the number of noun senses in WordNet plus the number of nouns in the document, to handle the possibility of words not found in WordNet. (This is the maximum size that could possibly be needed.) A metachain represents all possible chains that can contain the sense whose number is the index of the array. When a noun is encountered, for every sense of the noun in WordNet, the noun sense is placed into every metachain for which it has an identity, synonym, or hyperonym relation with that sense. These metachains represent every possible interpretation of the text. Note that each metachain has an index that is a WordNet sense number, so in a very real way, we can say that a chain has an "overriding sense." Table 1 shows the structure of such an array, with each row being a metachain based on the sense listed in the first column. In each node of a given metachain, appropriate pointers are kept to allow fast lookup. In addition, in association with each word, a list of the metachains to which it belongs is also kept (not shown in table).

The second step, finding the best interpretation, is accomplished by making a second pass through the source document. For each noun, each chain to which the noun belongs is examined and a determination is made, based on the type of relation and distance factors, as to which metachain the noun contributes to most. In the event of a tie, the higher sense number is used, since WordNet is organized with more specific concepts indexed with higher numbers. The noun is then deleted from all other metachains. Once all of the nouns have been processed, what is left is the interpretation whose score is maximum. From this interpretation, the best (highest-scoring) chains can be selected. The algorithm in its entirety is outlined in Table 2.

1 WordNet is available at $\langle\mathrm{http}: / /$ www.cogsci.princeton.edu/ wn $\rangle$.

2 The part-of-speech tagger we used is available from $\langle$ http://www.rt66.com/gcooke/SemanTag $\rangle$. 
Table 1

Example of metachains.

\begin{tabular}{cllll}
\hline Index & Meaning & Chain & & \\
\hline 0 & person & John & Machine & \\
1 & unit & Computer & IBM & \\
2 & device & Computer & Machine & IBM \\
3 & organization & Machine & IBM & \\
4 & unknown & IBM & & \\
$\vdots$ & & & & \\
$N$ & & & & \\
\hline
\end{tabular}

Note: Assume the sentences "John has a computer. The machine is an IBM." and that the nouns have the following senses (meanings): John (0), computer $(1,2)$, machine $(0,2,3)$, IBM $(1,2,3,4)$, and that words are put in a chain if they have an identity relation. This table then depicts the metachains after the first step.

Table 2

Basic linear-time lexical chains algorithm.

Step 1 For each noun instance

For each sense of the noun instance

Compute all scored metachains

Step 2 For each noun instance

For each metachain to which the noun belongs

Keep word instance in the metachain to which it contributes most Update the scores of each other metachain

\subsection{Scoring System}

Our scoring system allows different types of relations within a lexical chain to contribute to that chain differently. Further, our scoring system allows the distance between word instances in the original document to affect the word's contribution to a chain. Table 3 shows the scoring values used by our algorithm. These values were obtained through empirical testing, and although not optimal, appear to give good results.

Each noun instance is included in a chain because either it is the first noun instance to be inserted or it is related to some word that is already in the chain. If it is the first word, then the "identical word" relation score is used. If not, then the type of relation is determined, and the closest noun in the chain to which it is related is found. Using the distance between these two words and the relation type, we look up the contribution of the word instance to the overall chain score.

Once chains are computed, some of the high-scoring ones must be picked as representing the important concepts from the original document. To select these, we use the idea of "strong chains" introduced by Barzilay and Elhadad (1997). They define a strong chain as any chain whose score is more than two standard deviations above the mean of the scores computed for every chain in the document. 
Table 3

Scoring system tuned by empirical methods.

\begin{tabular}{lcccc}
\hline & One Sentence & Three Sentences & Same Paragraph & Default \\
\hline Identical word & 1 & 1 & 1 & 1 \\
Synonym & 1 & 1 & 1 & 1 \\
Hypernym & 1 & .5 & .5 & .5 \\
Sibling & 1 & .3 & .2 & 0 \\
\end{tabular}

Table 4

Constants from WordNet 1.6.

\begin{tabular}{lcc}
\hline Value & Worst Case & Average Case \\
\hline$C_{1}=\#$ of senses for a given word & 30 & 2 \\
$C_{2}=$ parent/child "is a" relations of a word sense & 45,147 & 14 \\
$C_{3}=\#$ of nouns in WordNet & 94,474 & 94,474 \\
$C_{4}=\#$ of synsets in WordNet & 66,025 & 66,025 \\
$C_{5}=\#$ of siblings of a word sense & 397 & 39 \\
$C_{6}=\#$ of chains to which a word instance can belong & 45,474 & 55
\end{tabular}

\section{Linear Runtime Proof}

In this analysis, we will not consider the computational complexity of part-of-speech tagging, since it is quite fast. The runtime of the full algorithm will be $O$ (pos tagging) $+O$ (our algorithm). Also, as it does not change from execution to execution of the algorithm, we shall take the size and structure of WordNet to be constant. We will examine each phase of our algorithm to show that the extraction of these lexical chains can indeed be performed in linear time. Table 4 defines constants for this analysis.

\subsection{Collection of WordNet Information}

For each noun in the source document that appears in WordNet, each sense that the word can take must be examined. Additionally, for each sense, we must walk up and down the hypernym/hyponym graph collecting all parent and child information. It is important to note that we are interested not only in direct parents and children, but in all parents and children in the graph from most specific to most general. Lastly we must collect all of the senses in WordNet that are siblings (i.e., share immediate parents) with the word being processed. All of the complexity in this step is related to the size of WordNet, which is constant. Lookups in WordNet use a binary search; hence a search in WordNet is $O\left(\log \left(C_{3}\right)\right)$. The runtime is given by

$$
n *\left(\log _{2}\left(C_{3}\right)+C_{1} * C_{2}+C_{1} * C_{5}\right) .
$$

\subsection{Building the Graph}

The graph of all possible interpretations is nothing more than an array of sense values $(66,025+n$ in size) that we will call the sense array. For each word, we examine each relation computed as above from WordNet. For each of these relations, we modify the list that is indexed in the sense array by the sense number of the noun's sense involved in the relation. This list is then modified by adding the word to the list and updating the list's associated score. Additionally, we add the chain's pointer (stored in the array) to a list of such pointers in the word object. Lastly, we add the value of 
how this word affects the score of the chain based on the scoring system to an array stored within the word structure. The runtime for this phase of the algorithm is

$$
n * C_{6} * 4,
$$

which is also clearly $O(n)$.

\subsection{Extracting the Best Interpretation}

For each word in the source document, we look at each chain to which the word can belong. A list of pointers to these chains is stored within the word object, so looking them up takes $O(1)$ time. For each of these, we simply look at the maximal score component value in all of these chains. We then set the scores of all of the nodes that did not contain the maximum to zero and update all the chain scores appropriately. The operation takes

$$
n * C_{6} * 4,
$$

which is also $O(n)$.

\subsection{Overall Runtime Performance}

The overall runtime performance of this algorithm is given by the sum of the steps listed above, for an overall runtime of

$$
\begin{gathered}
n *\left(1,548,216+\log _{2}(94,474)+45,474 * 4\right) \text { [worst case] } \\
n *\left(326+\log _{2}(94,474)+55 * 4\right) \text { [average case] } .
\end{gathered}
$$

Initially, we may be greatly concerned with the size of these constants; upon further analysis, however, we see that most synsets have very few parent-child relations. Thus the worst-case values may not reflect the actual performance of our application. In addition, the synsets with many parent-child relations tend to represent extremely general concepts such as "thing" and "object." These synsets will most likely not appear very often in a document.

Whereas in the worst case these constants are quite large, in the average case they are reasonable. This algorithm is $O(n)$ in the number of nouns within the source document. Considering the size of most documents, the linear nature of this algorithm makes it usable for generalized summarization of large documents (Silber and McCoy 2000). For example, in a test, our algorithm calculated a lexical chain interpretation of a 40,000-word document in 11 seconds on a Sparc Ultra 10 Creator. It was impossible to compute lexical chains for such a document under previous implementations because of computational complexity. Thus documents tested by Barzilay and Elhadad were significantly smaller in size. Our method affords a considerable speedup for these smaller documents. For instance, a document that takes 300 seconds using Barzilay and Elhadad's method takes only 4 seconds using ours (Silber and McCoy 2000).

\section{Evaluation Design}

Our algorithm now makes it feasible to use lexical chains as the method for identifying important concepts in a document, and thus they may now form the basis of an intermediate representation for summary generation, as proposed by Barzilay and Elhadad. An important consequence of this is that Barzilay and Elhadad's proposal can now be evaluated on documents of substantial size. We propose an evaluation of this intermediate stage that is independent of the generation phase of summarization. 
This said, we make no attempt to claim that a summary can actually be generated from this representation; we do attempt, however, to show that the concepts found in a human-generated summary are indeed the concepts identified by our lexical chains algorithm.

The basis of our evaluation is the premise that if lexical chains are a good intermediate representation for summary generation, then we would expect that each noun in a given summary should be used in the same sense as some word instance grouped into a strong chain in the original document on which the summary is based. Moreover, we would expect that all (most) strong chains in the document should be represented in the summary.

For this analysis, a corpus of documents with their human-generated summaries are required. Although there are many examples of document and summary types, for the purposes of this experiment, we focus on two general categories of summaries that are readily available. The first, scientific documents with abstracts, represents a readily available class of summaries often discussed in the literature (Marcu 1999). The second class of document selected was chapters from university level textbooks that contain chapter summaries. To prevent bias, textbooks from several fields were chosen.

In this analysis, we use the term concept to denote a noun in a particular sense (a given sense number in the WordNet database). It is important to note that different nouns with the same sense number ${ }^{3}$ are considered to be the same concept. It is also important to note that for the purposes of this analysis, when we refer to the "sense" of a word, we mean the sense as determined by our lexical chain analysis. The basic idea of our experiment is to try to determine whether the concepts represented by (strong) lexical chains in an original document appear in the summary of that document and whether the concepts appearing in the summary (as determined by the lexical chain analysis of the summary) come from strong chains in the document. If both of these give $100 \%$ coverage, this would mean that all and only the concepts identified by strong lexical chains in the document occur in the summary. Thus the higher these numbers turn out to be, the more likely it is that lexical chains are a good intermediate representation of the text summarization task.

A corpus was compiled containing the two specific types of documents, ranging in length from 2,247 to 26,320 words each. These documents were selected at random, with no screening by the authors. The scientific corpus consisted of 10 scientific articles (5 computer science, 3 anthropology, and 2 biology) along with their abstracts. The textbook corpus consisted of 14 chapters from 10 university level textbooks in various subjects (4 computer science, 6 anthropology, 2 history, and 2 economics), including chapter summaries.

For each document in the corpus, the document and its summary were analyzed separately to produce lexical chains. In both cases we output the sense numbers specified for each word instance as well as the overriding sense number for each chain. By comparing the sense numbers of (words in) each chain in the document with the computed sense of each noun instance in the summary, we can determine whether the summary indeed contains the same "concepts" as indicated by the lexical chains. For the analysis, the specific metrics we are interested in are

- The number and percentage of strong chains from the original text that are represented in the summary. Here we say a chain is represented if a

3 Recall that synonyms in the WordNet database are identified by a synset (sense) number. 
word occurs in the summary in the same sense as in the document strong chain. (Analogous to recall)

- The number and percentage of noun instances in the summary that represent strong chains in the document. (Analogous to precision)

By analyzing these two metrics, we can determine how well lexical chains represent the information that appears in these types of human-generated summaries. We will loosely use the terms recall and precision to describe these two metrics.

\subsection{Experimental Results}

Each document in the corpus was analyzed by running our lexical chain algorithm and collecting the overriding sense number of each strong lexical chain computed. Each summary in the corpus was analyzed by our algorithm, and the disambiguated sense (i.e., the sense of the noun instance that was selected in order to insert it into a chain) of each noun was collected. Table 5 shows the results of this analysis. The number of strong chains computed for the document is shown in column 2. Column 3 shows the

Table 5

Evaluation results.

\begin{tabular}{|c|c|c|c|c|}
\hline Document & $\begin{array}{l}\text { Total } \\
\text { Number } \\
\text { of Strong } \\
\text { Chains in } \\
\text { Document }\end{array}$ & $\begin{array}{c}\text { Total } \\
\text { Number } \\
\text { of Noun } \\
\text { Instances } \\
\text { in Summary }\end{array}$ & $\begin{array}{c}\text { Strong Chains } \\
\text { with } \\
\text { Corresponding } \\
\text { Noun Instances } \\
\text { in Summary }\end{array}$ & $\begin{array}{l}\text { Noun Instances } \\
\text { with } \\
\text { Corresponding } \\
\text { Strong Chains in } \\
\text { Document }\end{array}$ \\
\hline CS Paper 1 & 10 & 22 & $7 \quad(70.00 \%)$ & $19(86.36 \%)$ \\
\hline CS Paper 2 & 7 & 19 & $6 \quad(71.43 \%)$ & $17(89.47 \%)$ \\
\hline CS Paper 3 & 5 & 31 & $4 \quad(80.00 \%)$ & $27(87.19 \%)$ \\
\hline CS Paper 4 & 6 & 25 & $5 \quad(83.33 \%)$ & $24(96.00 \%)$ \\
\hline CS Paper 5 & 8 & 16 & $6 \quad(75.00 \%)$ & $12(75.00 \%)$ \\
\hline ANTH Paper 1 & 7 & 20 & $7(100.00 \%)$ & $17(85.00 \%)$ \\
\hline ANTH Paper 2 & 5 & 17 & $4 \quad(80.00 \%)$ & $13(76.47 \%)$ \\
\hline ANTH Paper 3 & 7 & 21 & $6 \quad(28.57 \%)$ & 7 (33.33\%) \\
\hline BIO Paper 1 & 4 & 19 & $4(100.00 \%)$ & $17(89.47 \%)$ \\
\hline BIO Paper 2 & 5 & 31 & $5 \quad(80.00 \%)$ & $28(90.32 \%)$ \\
\hline CS Chapter 1 & 9 & 55 & $8 \quad(88.89 \%)$ & $49(89.09 \%)$ \\
\hline CS Chapter 2 & 7 & 49 & $6 \quad(85.71 \%)$ & $42(85.71 \%)$ \\
\hline CS Chapter 3 & 11 & 31 & $9 \quad(81.82 \%)$ & $25(80.65 \%)$ \\
\hline CS Chapter 4 & 14 & 47 & $5 \quad(35.71 \%)$ & $21(44.68 \%)$ \\
\hline ANTH Chapter 1 & 5 & 61 & $4 \quad(80.00 \%)$ & $47(77.05 \%)$ \\
\hline ANTH Chapter 2 & 8 & 74 & $7 \quad(87.50 \%)$ & $59(79.73 \%)$ \\
\hline ANTH Chapter 3 & 12 & 58 & $11 \quad(91.67 \%)$ & $48(82.76 \%)$ \\
\hline ANTH Chapter 4 & 13 & 49 & $11 \quad(84.62 \%)$ & $42(85.71 \%)$ \\
\hline ANTH Chapter 5 & 7 & 68 & $5 \quad(71.43 \%)$ & $60(88.24 \%)$ \\
\hline ANTH Chapter 6 & 9 & 59 & $8 \quad(88.89 \%)$ & $48(81.36 \%)$ \\
\hline HIST Chapter 1 & 12 & 71 & $10 \quad(83.33 \%)$ & $67(94.37 \%)$ \\
\hline HIST Chapter 2 & 8 & 65 & $7 \quad(87.50 \%)$ & $55(84.62 \%)$ \\
\hline ECON Chapter 1 & 14 & 68 & $12 \quad(85.71 \%)$ & $63(92.65 \%)$ \\
\hline ECON Chapter 2 & 9 & 51 & $7 \quad(77.78 \%)$ & $33(64.71 \%)$ \\
\hline Mean & & & $79.12 \%$ & $80.83 \%$ \\
\hline Median & & & $82.58 \%$ & $85.35 \%$ \\
\hline
\end{tabular}

Note: ANTH—anthropology, BIO-biology, CS—computer science, ECON-economics, HIST-history. 
total number of noun instances found in the summary. Column 4 shows the number, and percentage overall, of strong chains from the document that are represented by noun instances in the summary (recall). The number, and the percentage overall, of nouns of a given sense from the summary that have a corresponding strong chain with the same overriding sense number (representing the chain) in the original text are presented in column 5 (precision). Summary statistics are also presented.

In $79.12 \%$ of the cases, lexical chains appropriately represent the nouns in the summary. In $80.83 \%$ of the cases, nouns in the summary would have been predicted by the lexical chains. The algorithm performs badly on two documents, anthropology paper 3 and computer science chapter 4, under this analysis. Possible reasons for this will be discussed below, but our preliminary analysis of these documents leads us to believe that they contain a greater number of pronouns and other anaphoric expressions (which need to be resolved to compute lexical chains properly). These potential reasons need to be examined further to determine why our algorithm performs so poorly on these documents. Excluding these two documents, our algorithm has a recall of $83.39 \%$ and a precision of $84.63 \%$ on average. It is important to note that strong chains represent only between $5 \%$ and $15 \%$ of the total chains computed for any document.

The evaluation presented here would be enhanced by having a baseline for comparison. It is not clear, however, what this baseline should be. One possibility would be to use straight frequency counts as an indicator and use these frequency counts for comparison.

\section{Discussion and Future Work}

Some problems that cause our algorithm to have difficulty, specifically proper nouns and anaphora resolution, need to be addressed. Proper nouns (people, organization, company, etc.) are often used in naturally occurring text, but since we have no information about them, we can only perform frequency counts on them. Anaphora resolution, especially in certain domains, is a bigger issue. Much better results are anticipated with the addition of anaphora resolution to the system.

Other issues that may affect the results we obtained stem from WordNet's coverage and the semantic information it captures. Clearly, no semantically annotated lexicon can be complete. Proper nouns and domain-specific terms, as well as a number of other words likely to be in a document, are not found in the WordNet database. The system defaults to word frequency counts for terms not found. Semantic distance in the "is a" graph, a problem in WordNet, does not affect our implementation, since we don't use this information. It is important to note that although our system uses WordNet, there is nothing specific to the algorithm about WordNet per se, and any other appropriate lexicon could be "plugged in" and used.

Issues regarding generation of a summary based on lexical chains need to be addressed and are the subject of our current work. Recent research has begun to look at the difficult problem of generating a summary text from an intermediate representation. Hybrid approaches such as extracting phrases instead of sentences and recombining these phrases into salient text have been proposed (Barzilay, McKeown, and Elhadad 1999). Other recent work looks at summarization as a process of revision; in this work, the source text is revised until a summary of the desired length is achieved (Mani, Gates, and Bloedorn 1999). Additionally, some research has explored cutting and pasting segments of text from the full document to generate a summary (Jing and McKeown 2000). It is our intention to use lexical chains as part of the input to a more classical text generation algorithm to produce new text that captures the concepts from the extracted chains. The lexical chains identify noun (or argument) concepts for the 
summary. We are examining ways for predicates to be identified and are concentrating on situations in which strong lexical chains intersect in the text.

\section{Conclusions}

In this article, we have outlined an efficient, linear-time algorithm for computing lexical chains as an intermediate representation for automatic machine text summarization. This algorithm is robust in that it uses the method proposed by Barzilay and Elhadad, but it is clearly $O(n)$ in the number of nouns in the source document.

The benefit of this linear-time algorithm is its ability to compute lexical chains in documents significantly larger than could be handled by Barzilay and Elhadad's implementation. Thus, our algorithm makes lexical chains a computationally feasible intermediate representation for summarization. In addition, we have presented a method for evaluating lexical chains as an intermediate representation and have evaluated the method using 24 documents that contain human-generated summaries. The results of these evaluations are promising.

An operational sample of our algorithm is available on the Web; a search engine that uses our algorithm can be accessed there as well (available at /http:/ /www.eecis. udel.edu/ silber/research.htm $\rangle$ ).

\section{Acknowledgments}

The authors wish to thank the Korean Government, Ministry of Science and Technology, whose funding, as part of the Bilingual Internet Search Machine Project, has made this research possible.

Additionally, special thanks to Michael Elhadad and Regina Barzilay for their advice and for generously making their data and results available, and to the anonymous reviewers for their helpful comments.

\section{References}

Barzilay, Regina and Michael Elhadad. 1997. Using lexical chains for text summarization. In Proceedings of the Intelligent Scalable Text Summarization Workshop (ISTS-97), Madrid, Spain.

Barzilay, Regina, Kathleen R. McKeown, and Michael Elhadad. 1999. Information fusion in the context of multi-document summarization. In Proceedings of the 37 th Annual Conference of the Association for Computational Linguistics, College Park, MD. Association for Computational Linguistics, New Brunswick, NJ.

Hirst, Graeme and David St.-Onge. 1997. Lexical chains as representation of context for the detection and correction of malapropisms. In Christiane Fellbaum, editor, Wordnet: An electronic lexical database and some of its applications. MIT Press, Cambridge, pages 305-332.

Jing, H. and K. McKeown. 2000. Cut and paste based text summarization. In Proceedings of NAACL-00, Seattle.

Mani, Inderjeet, Barbara Gates, and Eric Bloedorn. 1999. Improving summaries by revising them. In Proceedings of the 37 th Annual Conference of the Association for Computational Linguistics, College Park, MD. Association for Computational Linguistics, New Brunswick, NJ.

Marcu, Daniel. 1999. The automatic creation of large scale corpora for summarization research. In The 22nd International ACM SIGIR Conference on Research and Development in Information Retrieval, Berkeley. ACM Press, New York.

Morris, J. and G. Hirst. 1991. Lexical cohesion computed by thesaural relations as an indecator of the structure of text. Computational Linguistics, 18(1):21-45.

Silber, H. Gregory and Kathleen F. McCoy. 2000. Efficient text summarization using lexical chains. In 2000 International Conference on Intelligent User Interfaces, New Orleans, January.

Sparck Jones, Karen. 1993. What might be in summary? Information Retrieval '93, Regensburg, Germany, September, pages 9-26. 